\title{
Das Bild des Grafen Albert Joseph Hoditz (1706-1778) in der europäischen Literatur
}

\author{
Iveta $Z L A$
}

\begin{abstract}
The image of Count Albert Joseph Hoditz (1706-1778) in European literature

The article presents the image of Count Albert Joseph Hoditz (1706-1778) as depicted in European literature. The emphasis is not primarily on German-language literature; the study foregrounds the image of Count Hoditz in other European literatures. The investigation spans the period from the $19^{\text {th }}$ century to the beginning of the $21^{\text {st }}$ century.
\end{abstract}

Keywords: Rosswald, Silesia, Count Albert Joseph Hoditz, European literature, literary arrangement

DOI: doi.org/10.15452/StudiaGermanistica.2020.26.0008

\section{Einführung in die Thematik}

Graf Albert Joseph Hoditz beteiligte sich ebenso wie z. B. die Grafen Franz Anton von Sporck (1662-1738), Joseph Johann Maximilian Kinsky (1705-1780), Ignaz Dominik Chorynsky (1729-1792) und weitere adelige Repräsentanten der Kultur zwischen Barock und Aufklärung an der Herausbildung der künstlerischen Atmosphäre, die sowohl im Dienste der barocken Repräsentation stand, als auch sich durch die Durchsetzung von Idealen der Aufklärung auszeichnete. Albert Joseph Hoditz ist vor allem durch seine kulturellen Aktivitäten (vgl. Myška 2011), sein zwischen Genialität und Wahnsinn schwankendes Naturell sowie durch seine kulturellen Kontakte berühmt geworden. Der Adelshof dieses Adligen in Rosswald ${ }^{1}$ weckte das Interesse zahlreicher Reisender, Schriftsteller sowie Schauspieler. Diese Schlossresidenz wurde dank ihrer gartenarchitektonischen und kulturellen Vielfalt als „Rosswalder Arkadien“ und „Schlesisches Versailles“ bezeichnet. Der Ruf des Rosswalder Herrschaftsguts unter dem Grafen Albert Joseph Hoditz blieb auch einige Jahrhunderte nach seinem Tode bestehen und wurde in einigen Werken der europäischen schöngeistigen Literatur verewigt. Da den literarischen Darstellungen des Grafen Albert Joseph Hoditz in der deutschsprachigen Literatur bereits Aufmerksamkeit geschenkt wurde (vgl. Zlá 2015-2018, siehe Literaturverzeichnis), setzt sich die vorliegende Studie zum Ziel, seine Rolle in anderen europäischen Literaturen zu verdeutlichen. Diese Erforschung ist mit den Intentionen verbunden, auf die starke Resonanz des kulturellen Engagements von Hoditz im europäischen Maßstab hinzuweisen, seine Facetten zu verdeutlichen und die Rolle dieses Adligen in der europäischen Kultur anzudeuten.

Slezské Rudoltice, eine Gemeinde in der Nähe von Hotzenplotz (heute Osoblaha). 


\section{Der Graf Hoditz im literarischen Schaffen der tschechischen und slowa- kischen Autoren}

Mehr als sechs Jahrzehnte nach dem Tode des Grafen Albert Joseph Hoditz fanden seine kulturellen Ambitionen einen Nachhall in der tschechischen und slowakischen Literatur. Das Bild dieses Adligen taucht in den Werken der Autoren auf, die zur Zeit der Nationalen Wiedergeburt tätig waren. Das Phänomen Hoditz wird in die Atmosphäre der panslawischen Überlegungen eingebettet.

Der künstlerische Enthusiasmus des Grafen Albert Joseph Hoditz wird 1840 im Epigramm, Roswald‘ Josef Krasoslav Chmelenskýs (1800-1839) skizziert, das in seiner Gedichtsammlung ,Kwjtj polnj z Morawy a ze Slezka‘ (vgl. Chmelenský 1840:283 f.) 1840 erscheint. Die Epigramme werden durch Erfassungen der schlesischen Landschaft markiert, die verschiedene historische Affinitäten aufweisen. Einerseits wird der Ruf der Rosswalder arkadischen Gartenlandschaft hervorgehoben, andererseits wird er mit deren schlechtem Zustand nach dem Tode des Grafen Hoditz folgendermaßen kontrastiert:

Prw gsi šel Arkadij na Olymp sem, než Hodic usnul!

Ted' tebe tam poleká wandal i zřijceniny. ${ }^{2}$

1862 ist das Epos ,Slávy dcera ${ }^{3}$ des slowakischen Autors der Nationalen Wiedergeburt Ján Kollár (1793-1852) erschienen, in dem die Rosswalder Gartenszenerie mit der Hervorhebung der panslawischen Einstellung Kollárs verknüpft ist. Im zweiten Gesang dieses Epos erscheinen die Worte, die die Phänomenalität des Rosswalder Dominiums unterstreichen.

In den durch den umarmenden Reim gekennzeichneten Strophen wird dem Rosswalder Schlossgarten der inspirative Charakter verliehen. Das architektonische Gepräge der Rosswalder Gartenanlage wird in den Sonetten ${ }^{4}$ Kollárs im Spiegel seiner prachtvollen Schönheit dargestellt (vgl. Kollár 1852:279). Die im Epos thematisierte Gartenlandschaft ist als eine Verbindung der natürlichen Anmut mit der Gartenkunst dargestellt. Diese als „Paradies“ (vgl. Kollár 1852:279) aufgefasste Szenerie wird in der vierten Strophe des untersuchten Sonetts ${ }^{5}$ mit der gartenkünstlerischen Tätigkeit von Hoditz in Verbindung gebracht.

Obwohl die Widerspiegelung des Rosswalder Schlossgartens in einigen historisch (vgl. Myška 2011:10) sowie literaturgeschichtlich ausgerichteten Studien (vgl. Zlá 2015-2018, siehe Literaturverzeichnis) angedeutet wird, kommen in diesen Untersuchungen einige Ungenauigkeiten vor. In der historischen Forschungsliteratur taucht die These auf, nach der Rosswald im angeführten Epos als Konilesy (Myška 2011:10) bezeichnet wird. Der Rosswalder Schlossgarten wird jedoch im 146. Sonett dieses Epos als „Zahrad[a] konilesk[á]“ (vgl. Kollár 1852:197) thematisiert. Darüber hinaus belegt diese Textstelle, dass Rosswald nicht nur als „zahrady konileské“, sondern auch als „Rosvald“ bezeichnet wird. In der Schrift der Prager Königlichen Akademie der Wissenschaften (Královská společnost nauk 1876:240) wird das Toponymum „Konilesy“ lediglich mit den Überlegungen Ján Kollárs über dieses lyrisch-epische Gedicht (Kollár 1832) in Verbindung gebracht. In dieser Schrift sind jedoch keine Informationen über den literarischen Niederschlag der Rosswader Gartenlandschaft zu finden.

Der Graf Hoditz und sein „Schlesisches Versailles“ werden in der tschechischen bzw. tschechoslowakischen Literatur von den 70er Jahren des 19. Jh. bis in die 1950er Jahre kaum berührt. Eine Widerspiegelung des kulturellen Engagements von Hoditz taucht 1952 in der publizistischen Prosa

2 „Du gingst früher auf den Olymp durch die Arkadien, bevor Hoditz gestorben war, Jetzt bist du von Zerstörung und Ruinen erschrocken.“ (vgl. Chmelenský 1840:283 f.) Übersetzt von Iveta Zlá.

3 Im Katalog der Nationalbibliothek Wien ist das Epos als „Tochter des Ruhmes“ ins Deutsche übersetzt. Das Epos wurde jedoch auch als „Die Tochter der Slawa“ sowie „Die Tochter der Slava“ ins Deutsche übertragen.

4 Das Epos Slávy dcera besteht aus Sonetten. Die Studie stützt sich auf die vierte Ausgabe des Epos, die aus 645 Sonetten besteht. Die vierte Ausgabe des Epos ist 1852 erschienen.

5 Es handelt sich um die vierte Strophe des 126. Sonetts. (vgl. Kollár 1852:279) 
Karel Handzels ${ }^{6}$ auf, der den Grafen Albert Joseph Hoditz neben Petr Bezruč (1867-1958) und den Komponisten Pavel Kř́žzovský (1820-1885) stellt und sie mit dem fürstlichen Geist Schlesiens verbindet: „Bezruč, Kř́žkovský, hrabě Hodic v Rudolticích [...] jsou výrazem ,Knížecího Ducha Slezska. “"7

Das Rosswalder Herrschaftsgut und der Graf Albert Joseph Hoditz wurden 1992 im Essay Jiří Dahnes (1937-1999) ,Kouzelník z Rosswaldu“ (vgl. Daehne 1992:15-16) verewigt (vgl. Martínek 2009:7) und mit dem bewegten Leben sowie mit den kulturellen Ambitionen des Grafen Albert Joseph Hoditz verbunden.

2009 ist der Roman Blanka Jehlínkovás (*1963) ,Hrabě Albert Hodic. Blázen z Rudoltic‘ (Jehlínková 2009) erschienen, in dem der Graf Hoditz im Mosaik der Fabulierungskunst der Autorin angesiedelt ist. Die Romanhandlung spielt im Wirrwarr des Siebenjährigen Krieges und wird von einer Prophezeiung eingeleitet. Laut einer Wahrsagerin wird Hoditz weder Soldat noch Diplomat, dennoch erlangt er Berühmtheit. Der Graf Albert Joseph Hoditz wird als ein Kunstliebhaber dargestellt, dessen Leben permanent von finanziellen Problemen gebrandmarkt wird. Vor dem Hintergrund seiner Lebenseskapaden vollzieht sich die innere Entwicklung der zentralen Romanfigur, die sowohl in der Ehe mit Sophia Brandenburg-Bayreuth als auch in der Rosswalder kulturellen Plattform resultiert. Der Kontrast zwischen dem alles beherrschenden Krieg und der pazifistischen Einstellung des Grafen Hoditz zeigt sich u. a. in der Atmosphäre des Friedens und Glücks seines Dominiums. Die Autorin stellt in diesem Zusammenhang die Toleranz sowie den Kosmopolitismus des Grafen Hoditz in den Vordergrund.

Das Bild des Grafen Hoditz in der tschechischen und slowakischen Literatur ist mit der Hervorhebung des kulturellen Renommees der Rosswalder Schlossresidenz zu Lebzeiten des Grafen Albert Joseph Hoditz verknüpft. Sein Beitrag für die kulturelle Entwicklung Schlesiens wird mit dem Untergang der Rosswalder Gartenlandschaft nach dem Tode des Grafen Albert Joseph Hoditz kontrastiert, wodurch die Bedeutsamkeit der kulturellen Tätigkeit dieses Adligen unterstrichen wird.

\section{Graf Albert Joseph Hoditz in der französischen Literatur des Verklärung- srealismus}

Im reichhaltigen literarischen Schaffen der unter dem Pseudonym George Sand schreibenden französischen Autorin Amantine-Aurore-Lucile Dupin (1804-1876) wurde neben den sozialkritischen Momenten $^{8}$ die gesellschaftliche Stellung der Frau thematisiert. Die in der ersten Hälfte des 19. Jh. skandalös wirkenden Romane von Sand wie ,Indiana' (vgl. Sand 1832), ,Lélia‘ (vgl. Sand 1833), „Jeanne“ (vgl. Sand 1844), ,Consuelo‘ (vgl. Sand 1842-1843) etc. wurden vom Lesepublikum nicht nur positiv aufgenommen, sondern sie wurden auch der Kritik unterzogen.

Der Roman ,Consuelo' George Sands zeichnet die Laufbahn der begabten Sängerin Consuelo nach, die vom italienischen Komponisten Porpora musikalisch ausgebildet wird. Im Spiegel dieser Schilderung werden nicht nur verschiedene menschliche Charaktereigenschaften, Lebenseinstellungen und Schauplätze variiert, sondern die Romanhandlung enthüllt auch die vom Grafen Albert von Rudolstadt artikulierte Kritik der Ungerechtigkeit, Skrupellosigkeit und Unfreiheit in der zeitgenössischen Gesellschaft.

\footnotetext{
Karel Handzel hat in seinem literarischen Schaffen vor allem das Leben der mährisch-schlesischen Bergleute sowie die Sprache und Kultur in dieser Region thematisiert.

7 „Bezruč, Kř́žkovský, der Graf Hoditz in Rosswald [...] drücken den fürstlichen Geist Schlesiens aus.“ (Závodský 1954:76). Übersetzt von Iveta Zlá.

8 Der sozialen Problematik hat sich George Sand in ihren Romanen sowie in der Novelle ,Lavinia - Pauline - Cora von 1893 zugewendet. Sie ist auf diese Thematik auch in ihren Zeitungsstudien eingegangen. (vgl. Massardier-Kenney 1994:4).
} 
In dieses Romansujet hat die literarische Gestaltung des Grafen Albert Joseph Hoditz Eingang gefunden, ${ }^{9}$ der vor dem Hintergrund seiner Liebesbeziehung zu Sophie von Sachsen-Weißenfels thematisiert wird. Die von der Verherrlichung dieser Frau und einer sehr zärtlichen Anhänglichkeit (Krompotic 2007:378) durchdrungene Beziehung ist durch die gemeinsamen Interessen der Eheleute gekennzeichnet, die in ihrem Rosswalder Reich der Museen und Grazien (Krompotic 2007:378) umgesetzt werden. Auf die Einladung Consuelos nach Rosswald folgt ein Fest für die Gattin des Grafen Hoditz, das im künstlerischen Milieu des Rosswalder Dominiums stattfindet. Der Einblick in die Beziehung des Grafen Hoditz zu seiner Ehefrau sowie in die kulturelle Tätigkeit dieses Adligen wird von den Schilderungen der Rosswalder Elyseischen Felder (Krompotic 2007:378) begleitet. Sie werden von der zentralen Romanfigur sowohl für ihre Einbettung in die Natur bewundert, als auch für die geschmacklosen Verschönerungen moniert (vgl. Krompotic 2007:378).

Dennoch wurde der Graf Hoditz im untersuchten Roman als ein opferwilliger, gastfreundlicher und hochausgebildeter Adliger dargestellt, der die Ausbildung und moralische Vervollkommnung in den Vordergrund stellt.

Die Romanhandlung ist durch die Schilderungen des Rosswalder kulturellen Kolorits gekennzeichnet, die das kulturelle Engagement des Grafen Hoditz hervorheben. Darüber hinaus bietet die Romanhandlung einen Einblick in die Dramen- und Opernaufführungen im Rosswalder Schlosstheater, die zu Ehren der Markgräfin Sophia von Sachsen-Weißenfels veranstaltet wurden (vgl. Sand 1842-1843:515 f.).

Die literarische Gestaltung des Grafen Albert Joseph Hoditz im Roman ,Consuelo' George Sands ist als ein Kontrapunkt zum ungerechten, durch Kriege gebrandmarkten Weltbild zu betrachten. Sein Herrschaftsgut wird zu einem friedlichen, durch Festivitäten, Glück und Freude erfüllten Ort verwandelt, dessen Atmosphäre Ruhe, künstlerische Inspiration, Vergnügung und Belehrung bietet.

\section{Einblick in das kulturelle Engagement des Grafen Albert Joseph Hoditz in der Literatur der rumänischen Moderne}

Die Nachzeichnung der rumänischen Gesellschaft, Hervorhebung der Phantasie, bizarre Schilderungen des balkanischen Kolorits sowie die Bewunderung der rumänischen Aristokratie vom Anfang des 20. Jh. durchziehen das literarische Schaffen Mateiu Ion Caragiales (1885-1936). Obwohl sich sein literarisches Werk von den Gedichten mit mittelalterlicher Thematik (vgl. Caragiale 1936) nicht trennen lässt, ist er vor allem als Romancier (vgl. Caragiale 1929, ders. 1930-1933) und Novellenautor (vgl. Caragiale 1924) berühmt geworden. Sein literarisches Schaffen balanciert zwischen der Wirklichkeitsschilderung und Flucht in die Phantasiewelt, die er auch mit der Rosswalder Gartenlandschaft ${ }^{10}$ verband.

Im Roman ,Craii de Cuertea Veche‘ (vgl. URL 1) schildert der Erzähler die Phantasielandschaften, in die er sich mit seinen Bekannten begibt. Die Romanhandlung setzt 1910 in Bukarest

Obwohl George Sand Schlesien wahrscheinlich nie besucht hat, konnte sie Informationen über das „Schlesische Versailles“ des Grafen Albert Joseph Hoditz den Reisebeschreibungen des ausgehenden 18. Jh. entnehmen. Vgl. z. B. Comte de Guibert, Jacques Antoine Hippolyte Journal d'un voyage en Allemagne fait en 1773 par G.-A.-H. Guibert, ouvrage posthume publié par sa veuve et précédé d'une notice historique sur la vie de 1'auteur. Paris 1803. Die Hypothese über einen Aufenthalt dieser Autorin in Böhmen wurde in der Studie von Miroslav Prokop Haškovec aufgeworfen, dennoch wurde sie widerlegt. Die Reflexionen über die Beziehung George Sands zu Böhmen werden sowohl durch ihren Einfluss auf das literarische Schaffen der tschechischen Autorinnen des 19. Jh. wie Božena Němcová (1820-1862), Karolína Světlá (1830-1899) etc. sowie durch ihr Interesse für Persönlichkeiten wie Jan Hus (1369-1415), Jan Žižka (1360-1424) etc. untermauert (vgl. Haškovec 1925).

10 Obwohl Mateiu Ion Caragiale in seinem Roman ,Craii de Cuertea Veche“ den Rosswalder Schlossgarten schildert und den Grafen Albert Joseph Hoditz thematisiert, sind keine Informationen über seinen Einblick in die Biographie des Grafen Albert Joseph Hoditz überliefert. Er hat Rosswald wahrscheinlich nicht besucht. Er durfte die Informationen über den Grafen Hoditz während seines Berliner Studiums (1906-1908) bzw. zur Zeit seiner Anstellung als Leiter der Abteilung für Auslandspresse im Innenministerium Bukarest gewinnen. 
ein. Die thematisierten „Pilgerfahrten in die Phantasielandschaften“ sind durch die historische und geographische Entfernung gekennzeichnet. Vom Pazifischen Ozean über den Orient bis zum schlesischen Sitz des Grafen Albert Joseph Hoditz werden in die Romanhandlung zahlreiche Abenteuer, Begegnungen mit berühmten Persönlichkeiten sowie der Briefwechsel mit ihnen eingebunden.

Der Graf Albert Joseph Hoditz und sein Schlossgarten sind mit Verzauberung verknüpft, die die imaginären Reisenden dazu anregt, den Aufenthalt bei diesem Adligen zu verlängern. Dieser Ort der Museen und Grazien bietet ihnen einen freien Raum für die Entwicklung ihrer Vorstellungen und der Graf Hoditz wird kontextuell als Schöpfer eines Phantasiereiches betrachtet, mit dem die Romanfiguren die Vorliebe für das Phantastische und Unerwartete verbinden.

Die Darstellung des Grafen Albert Joseph Hoditz im untersuchten Roman Mateiu Ion Caragiales ist vor dem Hintergrund der mit der europäischen Literatur der Moderne zusammenhängenden Facetten zu betrachten. Sein literarisches Bild korrespondiert mit der Akzentuierung der Subjektivität sowie mit der von Konventionen befreiten Schöpfungskraft, die von den historischen, geographischen sowie sprachlichen Grenzen abstrahiert.

\section{Der Graf Albert Joseph Hoditz im Spiegel des Romans ,Il Fuoco‘ Gabriele D'Annunzios}

Das literarische Schaffen des italienischen Autors Gabriele D'Annunzio wurde nicht nur durch die Atmosphäre des Fin de Siècles geprägt, sondern auch von den spätromantischen und symbolistischen Merkmalen gekennzeichnet. Die Dominanz der Symbolik ist seinem Roman ,Il Fuoco' (vgl. D’Annunzio 1990) eigen, dessen Handlung in die Polarität des Feuers und Wassers eingespannt ist. Das Feuer ist mit dem Schöpfungsdrang und Vitalität verbunden, während das Wasser Leid und Tod repräsentiert. Das Alter Ego D’Annunzios wird im Roman von Stelio Effrena verkörpert, der als charismatischer Dichter berühmt geworden ist. Die Romanhandlung evoziert Reflexionen über die Botschaft der Liebe und Kunst sowie über die hinreißende und manipulierende Kraft der Sprache.

Im Roman wird die Figur des Fürsten Hoditz (D’Annunzio 1990:10) ins Mosaik der Überlegungen über die antike Kunst und germanische Mythologie platziert. Diese Romanfigur ist jedoch als eine der Nachkommen der Adelsfamilie von Hoditz zu verstehen, die sich nach der Umsetzung ihrer künstlerischen Ideen sehnt. Der Graf Albert Joseph Hoditz wird in die Romanhandlung als Kunst liebender Adliger eigebettet, dessen Schlossgarten zum Vorbild für die künstlerischen Vorhaben seines Nachkommen wird. Dieser Adlige wird als Schöpfer einer arkadischen Gartenlandschaft vorgestellt, in der er glückselig seine künstlerischen Visionen verwirklicht. (D’Annunzio 1990:10) Im Fokus der für die Romanhandlung kennzeichnenden Polarität des Feuers und Wassers sind die kulturellen Initiativen des Grafen Albert Joseph Hoditz als Inbegriff des durch die Kreativität geprägten Feuers zu betrachten. Sein Enthusiasmus ist auf die Vollendung seiner Märchenlandschaft ausgerichtet, die die Phantasien des Grafen widerspiegelt.

Obwohl das literarische Bild des Grafen Albert Joseph Hoditz im untersuchten Roman D'Annunzios lediglich auf die kulturellen Initiativen dieses Adligen eingeht, wird der literarische Nachhall seines Dominiums und seiner Lebensweise als Ausdruck der Kreativität und Tatkraft betrachtet.

\section{Schlussfolgerungen}

Die literarische Gestaltung des Grafen Albert Joseph Hoditz durchzieht das künstlerische Schaffen der tschechischen, slowakischen sowie französischen und italienischen Autoren seit der ersten Hälfte des 19. Jh., dringt in die Werke der rumänischen und italienischen Literatur vor und findet ebenfalls in der gegenwärtigen tschechischen Literatur Niederschlag. Das literarische Bild des Grafen Hoditz wird mit den thematischen Akzenten verbunden, die das künstlerische Engagement dieses Adligen in den Vordergrund rücken.

Die Erfassung des Grafen Hoditz in den untersuchten Werken lässt sich von der Darstellung seines Schlossgartens nicht trennen, der die Phantasien, Wünsche sowie hohe Ausbildung und 
Schöpfungskraft dieses Adligen widerspiegelt. Die prachtvolle Schönheit dieses Ortes wird von den Autoren der Nationalen Wiedergeburt als vorbildhaft besungen und mit ihrer späteren Zerstörung kontrastiert. Im kulturellen Klima der ersten Hälfte des 19. Jh. wird der Graf Albert Joseph Hoditz im Fokus des künstlerischen Enthusiasmus, der friedensstiftenden Ideen, der durch die Höflichkeit und Achtung geprägten Beziehung zu seiner Ehefrau sowie der Bemühungen um die Verbreitung der moralischen Prinzipien und Ausbildung dargestellt. Die durch die Impulse der Moderne inspirierten Romane Gabriele D'Annunzios und Mateiu Ion Caragiales akzentuieren die Kreativität des Grafen Albert Joseph Hoditz, dessen literarischer Niederschlag durch die Betonung der Phantasie und Freiheit gesteigert wird. In der tschechischen Literatur seit den 1990er Jahren werden biographische Momente ins Netz der Fabulierungskunst gesetzt.

Die Präsenz der mit dem Grafen Albert Joseph Hoditz verbundenen literarischen Variationen unterstreicht die Phänomenalität seines Wirkens und verdeutlicht seinen Nachhall.

\section{Literaturverzeichnis}

Caragiale, Mateiu (1930-1933): Sub pecetea tainei. Bukarest.

Caragiale, Mateiu Ion (1924): Remember. Bukarest.

Caragiale, Mateiu Ion (1929): Craii de Cuertea Veche. Bukarest.

Caragiale, Mateiu Ion (1936): Pajere. Bukarest.

Chmelenský, Josef Krasoslav (1840): Kwjtj polnj z Morawy a ze Slezka (Dokončenj). In: Maly, Jakub-Bohislav (Hrsg.): Dennice; spis zabawny. Praha, S. 283-284.

D’Annunzio, Gabriele (1900): Il Fuoco. Milano.

DAEHne, Jiří (1992): Kouzelník z Rosswaldu. In: Vlastivédné a kulturní neperiodikum Krnovsko. Editionsreihe II, Teil 3. Krnov, S. 15-16.

HAŠKoveC, Prokop Miroslav (1925): Byla George Sand v Čechách? Brno.

JeHLínKová, Blanka (2009): Hrabě Albert Hodic. Blázen z Rudoltic. Třebíč.

Kollár, Ján (1832): Wýklad čili Přjmětky a wysvětliwky ku Slávy Dceře. Pest.

Kollár, Ján (1852): Slávy dcera. Vierte Auflage. Praha.

KRÁLOVSKÁ SPOLEČNOST NAUK (Hrsg.) (1876): Zprávy o zasedání. Praha.

Krompotic, Louis (2007): Graf Albert Joseph von Hoditz-Roswald. In: SAND, George (Hrsg.): Consuelo. Bd. 8. Hannover, S. 378.

MARTíneK, Libor (2009): Mecenáš umění a svobodný zednář ze Slezských Rudoltic Albert Josef Hodic. In: Britské listy, S. 7.

Massardier-Kenney, Françoise (1994): Gender in the Fiction of George Sand. Amsterdam Atlanta.

MysKa, Milan (2011): Hrabě Hodic a jeho svět. Zámecká kultura ve Slezsku mezi barokem a osvícenstvim. Ostrava.

SAND, George (1832): Indiana. Paris.

SAND, George (1833): Lélia. Paris.

SAND, George (1842-1843): Consuelo. Paris.

SAND, George (1844): Jeanne. Paris.

ZÁvoDsKÝ, Artur (1954): Karel Handzel, spisovatel Ostravska. Praha.

ZlÁ, Iveta (2015): „Die Demut [kann] mich am sichersten führen.“ Die Widerspiegelung der Religiosität des Grafen Albert Joseph Hoditz (1706-1778) in seinem literarischen Nachlass. In: Brünner Beiträge zur Germanistik und Nordistik, Jg. 29, S. 127-135.

ZlÁ, Iveta (2015): Graf Albert Joseph Hoditz (1706-1778) im Spiegel der deutschsprachigen Literatur. In: Acta Facultatis Philosophicae Universitatis Ostraviensis Studia Germanistica, Nr. 17, Ostrava, S. 79-92.

ZLÁ, Iveta (2015): Literarische Gestaltung des Grafen Albert Joseph Hoditz (1706-1778) im Drama von Jakob Michael Reinhold Lenz „Der Tugendhafte Taugenichts“. In: Brünner Hefte zu 
Deutsch als Fremdsprache, Jg. 8, S. 45-54.

ZLÁ, Iveta (2016): Der literarische Nachlass des Grafen Albert Joseph Hoditz (1706-1778) im Fokus seiner Kontakte zum preußischen König Friedrich II. und zur österreichischen Kaiserin Maria Theresia. In: Germanoslavica. Zeitschrift für germano-slawische Studien, Nr. 2, S. 1-14.

ZLÁ, Iveta (2016): Literarische Gestaltung des Grafen Albert Joseph Hoditz (1706-1778) in der Novelle August Lewalds „Roswalde“. In: Brünner Beiträge zur Germanistik und Nordistik, S. 95-104.

ZLÁ, Iveta (2017): Der Graf Albert Joseph Hoditz (1706-1778) und das Rosswalder Dominium im Spiegel der Reisebeschreibung von Balthasar Ludwig Tralles „Schattenriss der Annehmlichkeiten von Roswalde“. In: Brünner Beiträge zur Germanistik und Nordistik, Nr. 31, S. 97-112.

Zlé, Iveta (2017): Graf Albert Joseph Hoditz als deutschsprachiger Autor. In: ACC JOURNAL, Nr. XXIII, S. 261-268.

ZLÁ, Iveta (2017): Literarische Affinitäten des Rosswalder Schlossgartens. In: Zentrum und Peripherie: Zentrum und Peripherie. Aus literaturwissenschaftlicher Sicht. Opava, S. 53-60.

ZLÁ, Iveta (2018): Lebensweise des Grafen Albert Joseph Hoditz im Spiegel seines literarischen Schaffens. In: Brünner Beiträge zur Germanistik und Nordistik, Nr. 32(2), S. 81-87.

\section{Internetquellen}

URL 1: http://www.seiten-der-dichtung.de/craii/vorbem_craii.htm/ [12.05.2020]. 